\title{
Palladium-Catalyzed Synthesis of Trimethylsilyl Substituted Benzopyran Derivatives
}

\author{
Yanmei Zhang ${ }^{1, *}, \quad$ Wei-Xing $\operatorname{Dan}^{1}$, Jianqi Liu ${ }^{1}$, Micky D. Tortorella ${ }^{1}$, Zhengchao $\mathrm{Tu}^{1}$ and \\ John J Talley ${ }^{2, *}$ \\ ${ }^{1}$ Drug Discovery Pipeline, Guangzhou Institute of Biomedicine and Health, Science City, Guangzhou, 510530, P. R. \\ China \\ ${ }^{2}$ Department of Chemistry, St. Louis University, St. Louis, MO 63108, USA
}

Abstract: Palladium catalysis is an efficient way to obtain trimethylsilyl substituted benzopyran derivatives. After screening a series of reactions, we found optimized conditions.

Keywords: Palladium-catalyzed, benzopyran, trimethyl silylation, selective COX-2 inhibitors.

\section{INTRODUCTION}

A number of benzopyran derivatives possess biological and pharmacological applications [1]. Studies show that benzopyrans have many biological activities such as antiestrogenic, insulin-sensitizing, selective thrombin inhibition, antimicrobial, antibacterial, hypoglycemic and so on [2-6]. Selected substituted $2 \mathrm{H}$-chromene-3-carboxylates were recently reported to possess anti-inflammatory and analgesic activities by selective inhibition of the inducible form of cyclooxygenase (COX-2) [7-9]. Among this series of compounds, the t-Bu substituted $2 \mathrm{H}$-chromene-3-carboxylates showed excellent selective inhibition of COX-2. Therefore there exists strong interest to develop trimethylsilyl substituted $2 \mathrm{H}$-chromene-3-carboxylates as $\mathrm{COX}-2$ inhibitors as a new chemical entity. In this study, we detail the preparation of trimethylsilyl substituted $2 \mathrm{H}$-chromene-3-carboxylates for evaluation as selective COX-2 inhibitors.

The most common method for synthesis of arylsilanes is the reaction of an aryllithium or magnesium reagent with halosilanes $[10,11]$. The discordancy of organometallic reagents with a number of aromatic substituents often dictate departure from this synthesis approach. To solve this problem, we sought a general synthesis method of arylsilanes compatible with a wide variety of functional groups, which was a strategy based on transition metal-catalyzed reaction of aryl halides with disilanes. Such a transformation was first described in a patent by Atwell and Bokermann in 1973 [12]. Subsequently, Matsumoto and co-workers prepared various arylsilanes from aryl bromides and selected electrondeficient aryl chlorides with hexamethyldisilane using $\mathrm{Pd}\left(\mathrm{PPh}_{3}\right)_{4}$ as catalyst [13-15]. Gooßen and co-workers disclosed a mild and efficient procedure for the catalytic silylation of aryl bromides in 2000 [16]. In spite of the aforementioned advancements, there remains considerable interest in the development of a robust general method for metalcatalyzed synthesis of arylsilanes.

In connection with our interest in the synthesis of $2 \mathrm{H}-$ chromene-3-carboxylate derivatives, we became interested in the synthesis of certain trimethylsilyl-2H-chromene-3carboxylates from their more readily available bromo- $2 \mathrm{H}$ chromene-3-carboxylate analogs by palladium catalysis (Scheme 1).<smiles>[R][R]1cc2c(cc1Br)C=C(C(=O)OCC)C(C(F)(F)F)O2</smiles>

Scheme (1). Palladium-catalyzed synthesis of trimethylsilyl-2H-chromene-3-carboxylate.

*Address correspondence to these authors at the Drug Discovery Pipeline, Guangzhou Institute of Biomedicine and Health, Science City, Guangzhou, 510530, P. R. China; Tel: +862032015277;

E-mail: zhang_yanmei@gibh.ac.cn; Department of Chemistry, St. Louis University, St. Louis, MO 63108, USA; E-mail: jtalley4@slu.edu

\section{RESULTS AND DISCUSSION}

Initially, we studied the reaction of aryl bromide (3a) with hexamethyldisilane as the model reaction for screening reaction conditions (Scheme 2, Table 1). To our delight, the desired product (4a) was isolated in high yield by employing 
the combination of $\mathrm{Pd}_{2} \mathrm{dba}_{3}, \quad$ [1,1'-biphenyl]-2-yldi-tertbutylphosphine, water and $\mathrm{KF}$ in tetrahydro-1,3-dimethyl$2(1 \mathrm{H})$ pyrimidone (DMPU) at $100{ }^{\circ} \mathrm{C}$ under argon (Table $\mathbf{1}$, entry 1). After screening other palladium catalysts, we found that $\mathrm{PdCl}_{2}(\mathrm{dppf}) \mathrm{CH}_{2} \mathrm{Cl}_{2}$ in the presence of [1,1'-biphenyl]-2yldi-tert-butylphosphine was a superior catalyst/ligand combination, producing $\mathbf{4 a}$ in even higher yield (Table 1, entries 1-4). The importance of added [1,1'-biphenyl]-2-yldi-tertbutylphosphine was illustrated in Table 1, entry 5 . No product was observed in the absence of [1,1'-biphenyl]-2-ylditert-butylphosphine. Encouraged by these results, we further optimized the reaction conditions by examination of experimental parameters including bases, solvents, ligands and reaction temperature (Table $\mathbf{1}$, entries $8-18$ ). No product (4a) was observed in the absence of bases (Table 1, entry 8). Of the bases tested: $\mathrm{KF}, \mathrm{K}_{2} \mathrm{CO}_{3}, \mathrm{~K}_{3} \mathrm{PO}_{4}, \mathrm{Cs}_{2} \mathrm{CO}_{3}$ (Table 1, entries 2, 9-11), KF consistently provided the most favorable yield of desired product. Among the solvents examined: DMPU, DMF, DMSO, and EtOH (Table 1, entries 2, 12-14),
DMPU was uniformly superior. Using our optimized catalyst system, solvent and base, we found that $100-120^{\circ} \mathrm{C}$ was optimal (Table 1, entries 16-18). We also examined the effect of the amount of $\mathrm{PdCl}_{2}(\mathrm{dppf}) \mathrm{CH}_{2} \mathrm{Cl}_{2}$, and found the amount of $\mathrm{PdCl}_{2}(\mathrm{dppf}) \mathrm{CH}_{2} \mathrm{Cl}_{2}$ was reduced in accordance with the decrease of target product yield (4a) (Table 1, entries 19-20). The yield of 4a significantly decreased when only 1.0 equiv of hexamethyldisilane was used (Table 1, entry 21) and the yield was also reduced when the amount of [1,1'-biphenyl]2-yldi-tert-butylphosphine was reduced (Table 1, entry 22). The yield of $\mathbf{4 a}$ decreased under anhydrous conditions (Table 1 , entry 23 versus entry 2 ). The optimum conditions were identified (Table 1, entry 2) affording 4a in $87 \%$ yield.

With optimized conditions in hand, the scope and limitations of this new methodology were evaluated as shown in Table 2. A variety of aryl bromides, possessing both electron-donating and electron-withdrawing substituents, were reacted with hexamethyldisilane to generate the arylsilanes

Table 1. Selected Results of Screening for Optimal Conditions

\begin{tabular}{|c|c|c|c|c|c|}
\hline Entry & Catalyst & Solvent & Base & Ligand & Yield (\%) \\
\hline 1 & $\mathrm{Pd}_{2} \mathrm{dba}_{3}$ & DMPU & $\mathrm{KF}$ & 2-(di-t-butylphosphino)biphenyl & 78 \\
\hline 2 & $\mathrm{PdCl}_{2}(\mathrm{dppf}) \mathrm{CH}_{2} \mathrm{Cl}_{2}$ & DMPU & $\mathrm{KF}$ & 2-(di-t-butylphosphino)biphenyl & 87 \\
\hline 3 & $\mathrm{Pd}\left(\mathrm{PPh}_{3}\right)_{4}$ & DMPU & $\mathrm{KF}$ & 2-(di-t-butylphosphino)biphenyl & 80 \\
\hline 4 & $\mathrm{PdCl}_{2}\left(\mathrm{PPh}_{3}\right)_{2}$ & DMPU & KF & 2-(di-t-butylphosphino)biphenyl & 56 \\
\hline 5 & $\mathrm{PdCl}_{2}(\mathrm{dppf}) \mathrm{CH}_{2} \mathrm{Cl}_{2}$ & DMPU & $\mathrm{KF}$ & none & NR \\
\hline 6 & $\mathrm{PdCl}_{2}(\mathrm{dppf}) \mathrm{CH}_{2} \mathrm{Cl}_{2}$ & DMPU & $\mathrm{KF}$ & tricyclohexyl phosphine & 28 \\
\hline 7 & $\mathrm{PdCl}_{2}(\mathrm{dppf}) \mathrm{CH}_{2} \mathrm{Cl}_{2}$ & DMPU & $\mathrm{KF}$ & $\mathrm{PPh}_{3}$ & $<5$ \\
\hline 8 & $\mathrm{PdCl}_{2}(\mathrm{dppf}) \mathrm{CH}_{2} \mathrm{Cl}_{2}$ & DMPU & none & 2-(di-t-butylphosphino)biphenyl & NR \\
\hline 9 & $\mathrm{PdCl}_{2}(\mathrm{dppf}) \mathrm{CH}_{2} \mathrm{Cl}_{2}$ & DMPU & $\mathrm{K}_{2} \mathrm{CO}_{3}$ & 2-(di-t-butylphosphino)biphenyl & 35 \\
\hline 10 & $\mathrm{PdCl}_{2}(\mathrm{dppf}) \mathrm{CH}_{2} \mathrm{Cl}_{2}$ & DMPU & $\mathrm{K}_{3} \mathrm{PO}_{4}$ & 2-(di-t-butylphosphino)biphenyl & $<5$ \\
\hline 11 & $\mathrm{PdCl}_{2}(\mathrm{dppf}) \mathrm{CH}_{2} \mathrm{Cl}_{2}$ & DMPU & $\mathrm{Cs}_{2} \mathrm{CO}_{3}$ & 2-(di-t-butylphosphino)biphenyl & $<5$ \\
\hline 12 & $\mathrm{PdCl}_{2}(\mathrm{dppf}) \mathrm{CH}_{2} \mathrm{Cl}_{2}$ & DMF & $\mathrm{KF}$ & 2-(di-t-butylphosphino)biphenyl & 79 \\
\hline 13 & $\mathrm{PdCl}_{2}(\mathrm{dppf}) \mathrm{CH}_{2} \mathrm{Cl}_{2}$ & DMSO & $\mathrm{KF}$ & 2-(di-t-butylphosphino)biphenyl & 47 \\
\hline 14 & $\mathrm{PdCl}_{2}(\mathrm{dppf}) \mathrm{CH}_{2} \mathrm{Cl}_{2}$ & $\mathrm{CH}_{3} \mathrm{CH}_{2} \mathrm{OH}$ & $\mathrm{KF}$ & 2-(di-t-butylphosphino)biphenyl & NR \\
\hline 15 & $\mathrm{PdCl}_{2}(\mathrm{dppf}) \mathrm{CH}_{2} \mathrm{Cl}_{2}$ & DMPU & $\mathrm{KF}$ & 2-(di-t-butylphosphino)biphenyl & $\mathrm{NR}^{\mathrm{b}}$ \\
\hline 16 & $\mathrm{PdCl}_{2}(\mathrm{dppf}) \mathrm{CH}_{2} \mathrm{Cl}_{2}$ & DMPU & $\mathrm{KF}$ & 2-(di-t-butylphosphino)biphenyl & $28^{\mathrm{c}}$ \\
\hline 17 & $\mathrm{PdCl}_{2}(\mathrm{dppf}) \mathrm{CH}_{2} \mathrm{Cl}_{2}$ & DMPU & $\mathrm{KF}$ & 2-(di-t-butylphosphino)biphenyl & $45^{\mathrm{d}}$ \\
\hline 18 & $\mathrm{PdCl}_{2}(\mathrm{dppf}) \mathrm{CH}_{2} \mathrm{Cl}_{2}$ & DMPU & $\mathrm{KF}$ & 2-(di-t-butylphosphino)biphenyl & $86^{\mathrm{e}}$ \\
\hline 19 & $\mathrm{PdCl}_{2}(\mathrm{dppf}) \mathrm{CH}_{2} \mathrm{Cl}_{2}$ & DMPU & $\mathrm{KF}$ & 2-(di-t-butylphosphino)biphenyl & $35^{\mathrm{f}}$ \\
\hline 20 & $\mathrm{PdCl}_{2}(\mathrm{dppf}) \mathrm{CH}_{2} \mathrm{Cl}_{2}$ & DMPU & $\mathrm{KF}$ & 2-(di-t-butylphosphino)biphenyl & $62^{\mathrm{g}}$ \\
\hline 21 & $\mathrm{PdCl}_{2}(\mathrm{dppf}) \mathrm{CH}_{2} \mathrm{Cl}_{2}$ & DMPU & $\mathrm{KF}$ & 2-(di-t-butylphosphino)biphenyl & $33^{\mathrm{h}}$ \\
\hline 22 & $\mathrm{PdCl}_{2}(\mathrm{dppf}) \mathrm{CH}_{2} \mathrm{Cl}_{2}$ & DMPU & $\mathrm{KF}$ & 2-(di-t-butylphosphino)biphenyl & $48^{\mathrm{i}}$ \\
\hline 23 & $\mathrm{PdCl}_{2}(\mathrm{dppf}) \mathrm{CH}_{2} \mathrm{Cl}_{2}$ & DMPU & $\mathrm{KF}$ & 2-(di-t-butylphosphino)biphenyl & $70^{\mathrm{j}}$ \\
\hline
\end{tabular}

${ }^{a}$ All reactions were run with aryl bromide (3a), hexamethyldisilane (2.0 equiv), Pd source (4.0 mol \%), ligand (15.0 mol \%), base (5.0 equiv), water(2.0 equiv), solvent $(10 \mathrm{~mL}), 100{ }^{\circ} \mathrm{C}$ under argon for 5 hours. Isolated yield. ${ }^{\mathrm{b}}$ at room temperature. ${ }^{\mathrm{c}}$ at $50{ }^{\circ} \mathrm{C} .{ }^{\mathrm{d}}$ at $80{ }^{\circ} \mathrm{C} .{ }^{\mathrm{e}}$ at $120^{\circ} \mathrm{C}$. ${ }^{\mathrm{f}} \mathrm{PdCl} \mathrm{Cl}_{2}(\mathrm{dppf}) \mathrm{CH}_{2} \mathrm{Cl} \mathrm{Cl}_{2}(2.0 \mathrm{~mol}$ \%). ${ }^{\mathrm{g}} \mathrm{PdCl}_{2}$ (dppf) $\mathrm{CH}_{2} \mathrm{Cl}_{2}$ ( $\left.3.0 \mathrm{~mol} \%\right) .{ }^{\mathrm{h}}$ hexamethyldisilane(1.0 equiv). ${ }^{\mathrm{i}}$-(di-t-butylphosphino)biphenyl $(10.0 \mathrm{~mol} \%) .{ }^{\mathrm{j}}$ no water. 
(4, Scheme 3). In general, we found substrates bearing electron donating substituent(s) afforded higher yields of desired silylated product, (Table 2, entries 8-14), whereas the yields were diminished slightly when electron- deficient aryl bromides were substrates, (Table 2, entries 4-7). Generally, 8aryl substituted derivatives gave higher yields than those substrates bearing an 8-position aliphatic moiety (Table 2 , entries $15,16,17$ vs $8,9,10,11,12,14)$. The steric effect the reaction system was further examined (Table 2, entries 1 , 2,3 vs 18) and found there was no silylation of aryl bromide (3r) with hexamethyldisilane due to methyl group at the oposition of reacting bromide.
Finally, the optimum conditions for saponification of the present trimethylsilyl substituted esters was found to be lithium hydroxide in mixture of methanol and water $\left(\mathrm{CH}_{3} \mathrm{OH}: \mathrm{H}_{2} \mathrm{O}=10: 1\right)$. All of the esters (4) were converted to the corresponding acids (5) in excellent yield under these conditions. With an ample supply of the desired compounds in hand their activity against cyclooxygenase- 2 was evaluated and will be reported upon in due course as further optimizations are made.

In summary, we have developed a general and efficient palladium-catalyzed silylation reaction of aryl $\mathrm{C}-\mathrm{Br}$ bonds, providing arylsilanes products in moderate to good yields.<smiles>CCOC(=O)C1=Cc2cc(Br)ccc2OC1C(F)(F)F</smiles>

$3 a$

$4 a$

Scheme (2). The synthesis of ethyl 2-(trifluoromethyl)-6-(trimethylsilyl)-2H-chromene-3 -carboxylate.

Table 2. Pd-Catalyzed Silylation of Aryl Bromides with Hexamethyldisilane

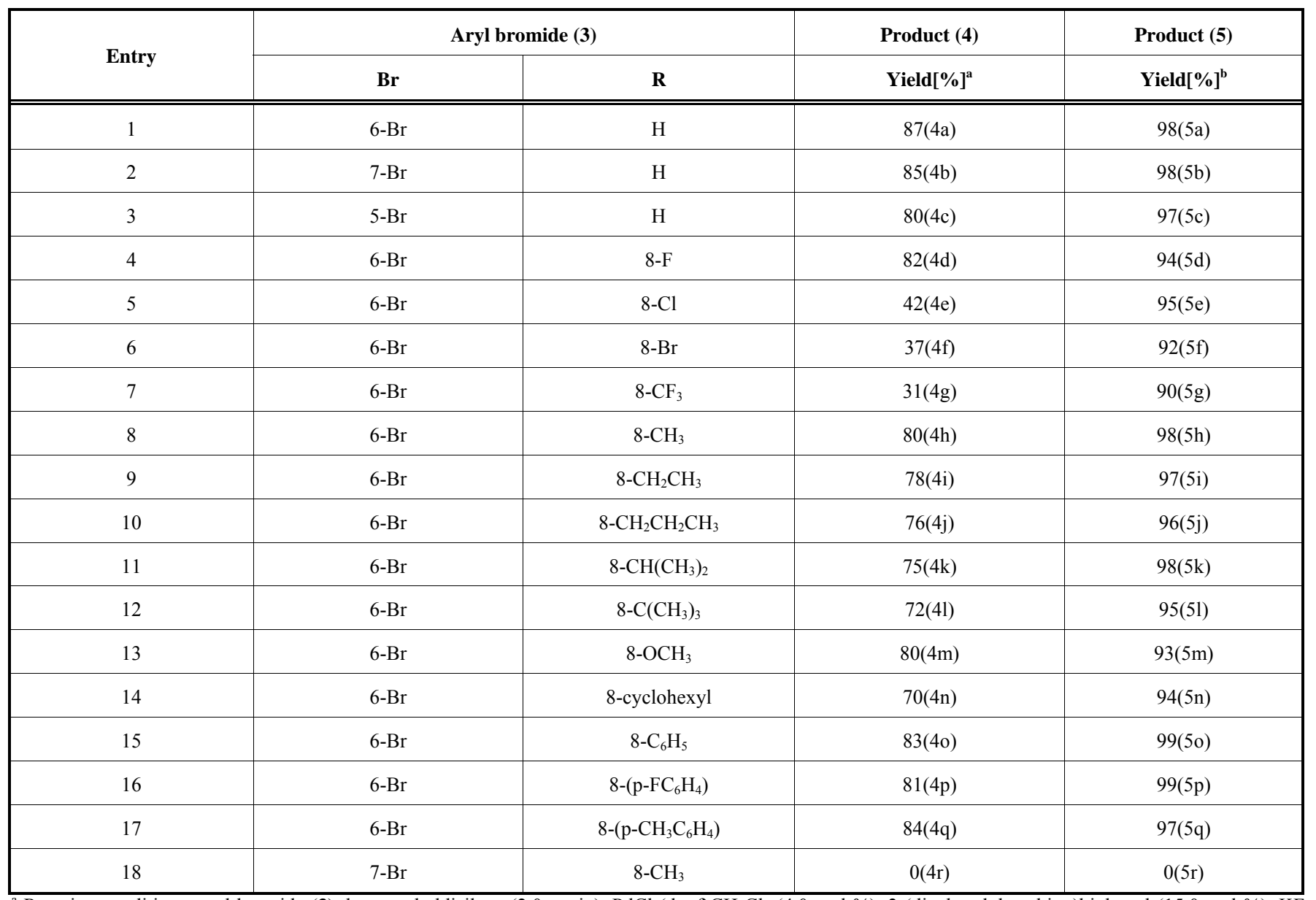

${ }^{a}$ Reaction conditions: aryl bromide (3), hexamethyldisilane (2.0 equiv), $\mathrm{PdCl}_{2}(\mathrm{dppf}) \mathrm{CH}_{2} \mathrm{Cl}_{2}(4.0 \mathrm{~mol} \%$ ), 2-(di-t-butylphosphino)biphenyl (15.0 mol \%), $\mathrm{KF}$ (5.0 equiv), water (2.0 equiv), DMPU $(10 \mathrm{~mL}), 100^{\circ} \mathrm{C}$ under argon for 5-8 hours. Isolated yield.

${ }^{b}$ Reaction conditions: arylsilanes (4), $\mathrm{LiOH}$ (10 equiv), solvent $\left(\mathrm{H}_{2} \mathrm{O}: \mathrm{EtOH}=1: 10\right)$, room temperature ,overnight, dilution hydrochloric acid; isolated yield. 


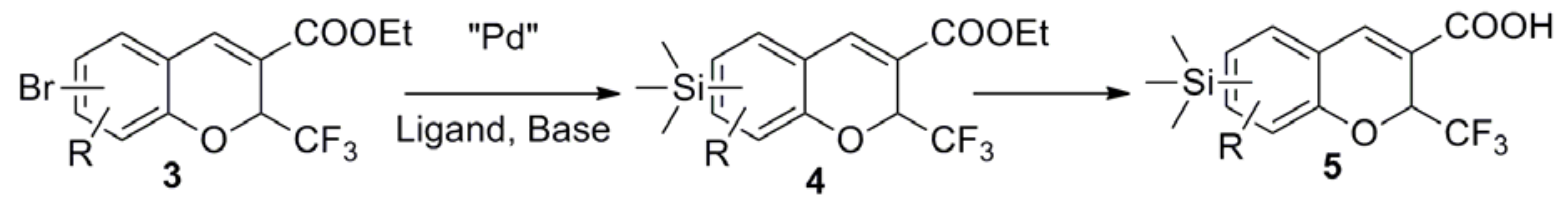

Scheme (3). Pd-Catalyzed Silylation of Aryl Bromides with hexamethyldisilane.<smiles>[R]c1cc(Br)ccc1OCCOCc1cc(Br)cc([R])c1O</smiles><smiles>[R]c1cc([Si](C)(C)C)cc2c1OC(C(F)(F)F)C(C(=O)OCC)=C2</smiles>

4<smiles>[R]c1cc([Si](C)(C)C)cc2c1OC(C(F)(F)F)C(C(=O)O)=C2</smiles>

5

Scheme (4). The design of compound synthesis.

Particularly noteworthy is the ability to conduct the silylation procedure without harm to the ethyl $2 \mathrm{H}$-chromene-3carboxylate ring.

Procedure for Synthesis of Trimethylsilyl Substituted Benzopyran Derivatives Synthesis of 5-bromo-2hydroxybenzaldehyde (2a, Scheme 4)

A stirred solution of 4-bromophenol $(3.81 \mathrm{~g}, 22.0 \mathrm{mmol})$ in trifluoroacetic acid $(50 \mathrm{~mL})$, was added hexamethylenetetramine $(3.70 \mathrm{~g}, 26.4 \mathrm{mmol})$ and maintained at $70^{\circ} \mathrm{C}$ for 2 h. After completion of the reaction (TLC), the mixture was quenched with water $(70 \mathrm{~mL})$ whereupon a solid precipitated. The mixture was stirred for $20 \mathrm{~min}$ and allowed to stand for $1 \mathrm{~h}$. The solid was isolated by filtration and washed with water. The solid was purified by column chromatography (petroleum ether/ethyl acetate, 100:1) to give title compound $(1.59 \mathrm{~g}, 36 \%)$.

${ }^{1} \mathrm{H}$ NMR $\left(\mathrm{CDCl}_{3}, 400 \mathrm{MHz}\right) \delta 6.91(\mathrm{~d}, J=8.8 \mathrm{~Hz}, 1 \mathrm{H})$, $7.60\left(\mathrm{dd}, J_{1}=2.4 \mathrm{~Hz}, J_{2}=8.8 \mathrm{~Hz}, 1 \mathrm{H}\right), 7.67(\mathrm{~d}, J=2.4 \mathrm{~Hz}, 1 \mathrm{H})$, $9.84(\mathrm{~s} 1 \mathrm{H}), 10.92(\mathrm{~s}, 1 \mathrm{H})$.

MS (MM-ES+APCI), m/z: 200 (M - 1).

Synthesis of ethyl 6-bromo-2-(trifluoromethyl)-2Hchromene-3-carboxylate (3a).

To a stirred solution of 5-bromo-2-hydroxybenzaldehyde $(1.59 \mathrm{~g}, 7.9 \mathrm{mmol})$, cesium carbonate $(2.61 \mathrm{~g}, 7.9 \mathrm{mmol})$ in dry $N, N$-dimethylformamide $(15 \mathrm{~mL})$, was added ethyl 4,4,4-trifluorocrotonate $(2.66 \mathrm{~g}, 15.8 \mathrm{mmol})$. The solution was warmed to $120^{\circ} \mathrm{C}$ for $4 \mathrm{~h}$, after completion of the reaction (MS), the mixture was quenched with water $(50 \mathrm{~mL})$, and extracted with ethyl acetate. The extract was dried over $\mathrm{Na}_{2} \mathrm{SO}_{4}$, concentrated in vacuo, and purified by column chromatography (petroleum ether/ethyl acetate, 20:1) to give title compound $(1.57 \mathrm{~g}, 57 \%)$.
${ }^{1} \mathrm{H}$ NMR $\left(\mathrm{CDCl}_{3}, 400 \mathrm{MHz}\right) \delta 7.64(\mathrm{~s} 1 \mathrm{H}), 7.40(\mathrm{dd}$, $J=1.6,8.4 \mathrm{~Hz}, 1 \mathrm{H}), 7.35(\mathrm{~d}, J=1.6 \mathrm{~Hz}, 1 \mathrm{H}), 6.87(\mathrm{~d}, J=8.4$ $\mathrm{Hz}, 1 \mathrm{H}), 5.70(\mathrm{q}, J=6.8 \mathrm{~Hz}, 1 \mathrm{H}), 4.32(\mathrm{q}, J=7.2 \mathrm{~Hz}, 2 \mathrm{H})$, $1.35(\mathrm{t}, J=7.2 \mathrm{~Hz}, 3 \mathrm{H})$.

MS (MM-ES+APCI), m/z: 350 (M - 1).

Synthesis of ethyl 2-(trifluoromethyl)-6-(trimethylsilyl)2H-chromene-3-carboxylate (4a).

To a solution of ethyl 6-bromo-2-(trifluoromethyl)-2Hchromene-3-carboxylate $(0.99 \mathrm{~g}, 2.8 \mathrm{mmol}), \mathrm{dba}_{3} \mathrm{Pd}_{2}(95.5$ $\mathrm{mg}, 0.11 \mathrm{mmol}), 2$-(di-t-butylphosphino)biphenyl (121.6 mg, $0.42 \mathrm{mmol})$, KF $(0.82 \mathrm{~g}, 14.0 \mathrm{mmol})$ in tetrahydro-1,3dimethyl-2(1H)pyrimidone $(10 \mathrm{~mL})$ under argon was added water $(100.8 \mathrm{mg}, 5.6 \mathrm{mmol})$, hexamethyldisilane $(0.68 \mathrm{~g} .5 .6$ $\mathrm{mmol}$ ) and the mixture was warmed at $100{ }^{\circ} \mathrm{C}$ for $4 \mathrm{~h}$. After completion of the reaction (MS), the mixture was quenched with water $(30 \mathrm{~mL})$, extracted with ethyl acetate. The organic layer was dried over $\mathrm{Na}_{2} \mathrm{SO}_{4}$, filtered, concentrated in vacuo, and purified by the column chromatography (petroleum ether/ethyl acetate, $30: 1)$ to get the title compound $(0.84 \mathrm{~g}$, $87 \%)$.

${ }^{1} \mathrm{H}$ NMR $\left(\mathrm{CDCl}_{3}, 400 \mathrm{MHz}\right) \delta 7.77(\mathrm{~s} 1 \mathrm{H}), 7.46(\mathrm{dd}, J=1.6$, $J=8.0 \mathrm{~Hz}, 1 \mathrm{H}), 7.36(\mathrm{~d}, J=1.6 \mathrm{~Hz}, 1 \mathrm{H}), 6.96(\mathrm{~d}, J=8.0 \mathrm{~Hz}$, $1 \mathrm{H}), 5.73(\mathrm{q}, J=6.8 \mathrm{~Hz}, 1 \mathrm{H}), 4.32(\mathrm{q}, J=7.2 \mathrm{~Hz}, 2 \mathrm{H}), 1.35(\mathrm{t}$, $J=7.2 \mathrm{~Hz}, 3 \mathrm{H}), 0.26$ (s, 9H).

MS (MM-ES+APCI), m/z: 344 (M - 1).

Synthesis of 2-(trifluoromethyl)-6-(trimethylsilyl)-2 $\mathrm{H}$ chromene-3-carboxylic acid (5a).

A mixture of ethyl 2-(trifluoromethyl)-6-(trimethylsilyl)$2 \mathrm{H}$-chromene-3-carboxylate $(0.75 \mathrm{~g}, 2.4 \mathrm{mmol})$, lithium hydroxide $(1.01 \mathrm{~g}, 24.0 \mathrm{mmol})$ was dissolved in methanol (20 $\mathrm{mL})$, water $(2 \mathrm{~mL})$, and then the mixture was stirred over- 
night at room temperature. After completion of the reaction (TLC), the mixture was concentrated in vacuo. The residue was stirred with dilute hydrochloric acid $(0.1 \mathrm{~mol} / \mathrm{L}, \mathrm{pH} 3)$, and then a white solid was formed, isolated washed and concentrated in vacuo, affording $743 \mathrm{mg}, 98 \%$ of compound 5 a.

White solid; mp 124-126 ${ }^{\circ} \mathrm{C} ;{ }^{1} \mathrm{H}$ NMR $\left(d_{6}\right.$-DMSO, 400 $\mathrm{MHz}) \delta 7.62$ (d, $J=1.6 \mathrm{~Hz}, 1 \mathrm{H}), 7.85$ (s, 1H), 7.50 (dd, $J=1.2$, $8.0 \mathrm{~Hz}, 1 \mathrm{H}), 7.02(\mathrm{~d}, J=8.0 \mathrm{~Hz}, 1 \mathrm{H}), 5.89$ (q, $J=7.2 \mathrm{~Hz}, 1 \mathrm{H})$, 0.23 (s, 9H); HRMS calcd for $\mathrm{C}_{14} \mathrm{H}_{15} \mathrm{~F}_{3} \mathrm{O}_{3} \mathrm{Si} \mathrm{m} / \mathrm{z}$ 317.08153, found 317.08194 .

2-(trifluoromethyl)-7-(trimethylsilyl)-2H-chromene-3carboxylic acid (5b): Yield 98\%; white solid; mp 102-104 ${ }^{\circ} \mathrm{C}$; 1H NMR (d6-DMSO, $\left.400 \mathrm{MHz}\right) \delta 0.23$ (s, 9H), 5.89 (q, $\mathrm{J}=7.2 \mathrm{~Hz}, 1 \mathrm{H}), 7.10(\mathrm{~s}, 1 \mathrm{H}), 7.18(\mathrm{~d}, \mathrm{~J}=7.2 \mathrm{~Hz}, 1 \mathrm{H}), 7.44(\mathrm{t}$, $\mathrm{J}=7.2 \mathrm{~Hz}, 1 \mathrm{H}), 7.84(\mathrm{~s}, 1 \mathrm{H})$; HRMS calcd for $\mathrm{C}_{14} \mathrm{H}_{15} \mathrm{~F}_{3} \mathrm{O}_{3} \mathrm{Si}$ $\mathrm{m} / \mathrm{z} 317.08153$, found 317.08182 .

2-(trifluoromethyl)-5-(trimethylsilyl)-2H-chromene-3carboxylic acid (5c): Yield 97\%; white solid; mp 202-203 ${ }^{\circ} \mathrm{C}$; 1H NMR (d6-DMSO, $\left.400 \mathrm{MHz}\right) \delta 0.33$ (s, 9H), 5.89 (q, $\mathrm{J}=7.2 \mathrm{~Hz}, 1 \mathrm{H}), 7.06(\mathrm{~d}, \mathrm{~J}=8.0 \mathrm{~Hz}, 1 \mathrm{H}), 7.19(\mathrm{~d}, \mathrm{~J}=7.2 \mathrm{~Hz}, 1 \mathrm{H})$, 7.37 (t, J=7.6Hz, 1H), 7.91 (s, 1H), 13.37 (brs, 1H); HRMS calcd for $\mathrm{C}_{14} \mathrm{H}_{15} \mathrm{~F}_{3} \mathrm{O}_{3} \mathrm{Si} \mathrm{m} / \mathrm{z} 317.08153$, found 317.08165 .

8-fluoro-2-(trifluoromethyl)-6-(trimethylsilyl)-2Hchromene-3-carboxylic acid (5d): Yield 94\%; white solid; mp 186-187 ${ }^{\circ} \mathrm{C}$; $\left.1 \mathrm{H} \mathrm{NMR} \mathrm{(d6-DMSO,} 400 \mathrm{MHz}\right) \delta 0.24$ (s, 9H), 6.06 (q, J=7.2Hz, 1H), 7.43-7.48 (m, 2H), $7.94(\mathrm{~s}, 1 \mathrm{H})$, 13.41 (brs, 1H); HRMS calcd for $\mathrm{C}_{14} \mathrm{H}_{14} \mathrm{~F}_{4} \mathrm{O}_{3} \mathrm{Si} \mathrm{m} / \mathrm{z}$ 335.07211 , found 335.07230 .

8-chloro-2-(trifluoromethyl)-6-(trimethylsilyl)-2Hchromene-3-carboxylic acid (5e): Yield 95\%; white solid; mp 199-201 ${ }^{\circ} \mathrm{C}$; $1 \mathrm{H}$ NMR (d6-DMSO, $\left.400 \mathrm{MHz}\right) \delta 0.24$ (s, 9H), 6.09 (q, J=7.2Hz, 1H), 7.56(s, 1H), $7.61(\mathrm{~s}, 1 \mathrm{H}), 7.90$ (s, 1H); HRMS calcd for $\mathrm{C}_{14} \mathrm{H}_{14} \mathrm{ClF}_{3} \mathrm{O}_{3} \mathrm{Si} \mathrm{m} / \mathrm{z} 351.04256$, found 351.04279 .

8-bromo-2-(trifluoromethyl)-6-(trimethylsilyl)-2Hchromene-3-carboxylic acid (5f): Yield 92\%; white solid; mp 204-206 ${ }^{\circ} \mathrm{C}$; $\left.1 \mathrm{H} \mathrm{NMR} \mathrm{(d6-DMSO,} 400 \mathrm{MHz}\right) \delta 0.25$ (s, 9H), 6.09 (q, J=7.2Hz, 1H), 7.65-7.69 (m, 2H), 7.90 (s, 1H), 13.38 (brs, $1 \mathrm{H}$ ); HRMS calcd for $\mathrm{C}_{14} \mathrm{H}_{14} \mathrm{BrF}_{3} \mathrm{O}_{3} \mathrm{Si} \mathrm{m} / \mathrm{z}$ 394.99204, found 394.99218.

2,8-bis(trifluoromethyl)-6-(trimethylsilyl)-2Hchromene-3-carboxylic acid (5g): Yield 90\%; pale yellow solid; mp 166-167 ${ }^{\circ} \mathrm{C}$; $1 \mathrm{H}$ NMR (d6-DMSO, $\left.400 \mathrm{MHz}\right) \delta$ 0.27 (s, 9H), 6.12 (q, J=7.2Hz, 1H), 7.71 (s, 1H), 7.97-7.98 (m, 2H); HRMS calcd for $\mathrm{C}_{15} \mathrm{H}_{14} \mathrm{~F}_{6} \mathrm{O}_{3} \mathrm{Si} \mathrm{m} / \mathrm{z} 385.06891$, found 385.06765 .

8-methyl-2-(trifluoromethyl)-6-(trimethylsilyl)-2Hchromene-3-carboxylic acid (5h): Yield 98\%; white solid; mp 163-165 ${ }^{\circ} \mathrm{C}$; $1 \mathrm{H}$ NMR (d6-DMSO, $\left.400 \mathrm{MHz}\right) \delta 0.23(\mathrm{~s}$, 9H), 2.19 (s, 3H), 5.94 (q, J=7.2Hz, 1H), 7.38 (s, 1H), 7.45 $(\mathrm{s}, 1 \mathrm{H}), 7.85(\mathrm{~s}, 1 \mathrm{H})$; HRMS calcd for $\mathrm{C}_{15} \mathrm{H}_{17} \mathrm{~F}_{3} \mathrm{O}_{3} \mathrm{Si} \mathrm{m} / \mathrm{z}$ 331.09718 , found 331.09727.

8-ethyl-2-(trifluoromethyl)-6-(trimethylsilyl)-2Hchromene-3-carboxylic acid (5i): Yield 97\%; white solid; mp 130-132 ${ }^{\circ} \mathrm{C}$; $1 \mathrm{H}$ NMR (d6-DMSO, $\left.400 \mathrm{MHz}\right) \delta 0.23$ (s, $9 \mathrm{H}), 1.14(\mathrm{t}, 3 \mathrm{H}), 2.54-2.67(\mathrm{~m}, 2 \mathrm{H}), 5.94(\mathrm{q}, \mathrm{J}=7.2 \mathrm{~Hz}, 1 \mathrm{H})$, $7.36(\mathrm{~s}, 1 \mathrm{H}), 7.44-7.45(\mathrm{~m}, 1 \mathrm{H}), 7.82(\mathrm{~s}, 1 \mathrm{H})$; HRMS calcd for $\mathrm{C}_{16} \mathrm{H}_{19} \mathrm{~F}_{3} \mathrm{O}_{3} \mathrm{Si} \mathrm{m} / \mathrm{z} 345.11283$, found 345.11323.
8-propyl-2-(trifluoromethyl)-6-(trimethylsilyl)-2Hchromene-3-carboxylic acid (5j): Yield 96\%; white solid; mp 175-177 ${ }^{\circ} \mathrm{C}$; $1 \mathrm{H}$ NMR (d6-DMSO, $\left.400 \mathrm{MHz}\right) \delta 0.23(\mathrm{~s}$, $9 \mathrm{H}), 0.89$ (t, J=7.2Hz, 3H), 1.51-1.60 (m, 2H), 2.57-2.64 (m, 2H), $5.92(\mathrm{q}, \mathrm{J}=7.2 \mathrm{~Hz}, 1 \mathrm{H}), 7.35(\mathrm{~m}, 1 \mathrm{H}), 7.45(\mathrm{~s}, 1 \mathrm{H}), 7.85$ (s, 1H) ; HRMS calcd for $\mathrm{C}_{17} \mathrm{H}_{21} \mathrm{~F}_{3} \mathrm{O}_{3} \mathrm{Si} \mathrm{m} / \mathrm{z} 359.12880$, found 359.12848 .

8-isopropyl-2-(trifluoromethyl)-6-(trimethylsilyl)-2Hchromene-3-carboxylic acid (5k): Yield 98\%; white solid; mp 140-142 ${ }^{\circ} \mathrm{C}$; $1 \mathrm{H}$ NMR (d6-DMSO, $400 \mathrm{MHz}$ ) $\delta 0.22$ (s, $9 \mathrm{H}), 1.19(\mathrm{dd}, \mathrm{J} 1=2.4 \mathrm{~Hz}, \mathrm{~J} 2=6.8 \mathrm{~Hz}, 6 \mathrm{H}), 3.17-3.24(\mathrm{~m}, 1 \mathrm{H})$, $5.92(\mathrm{q}, \mathrm{J}=7.2 \mathrm{~Hz}, 1 \mathrm{H}), 7.38-7.43(\mathrm{~m}, 2 \mathrm{H}), 7.83(\mathrm{~s}, 1 \mathrm{H})$; HRMS calcd for $\mathrm{C}_{17} \mathrm{H}_{21} \mathrm{~F}_{3} \mathrm{O}_{3} \mathrm{Si} \mathrm{m} / \mathrm{z}$ 359.12874, found 359.12848 .

8-(tert-butyl)-2-(trifluoromethyl)-6-(trimethylsilyl)-2Hchromene-3-carboxylic acid (5I): Yield 95\%; white solid; mp 174-175 ${ }^{\circ} \mathrm{C}$; $1 \mathrm{H}$ NMR (d6-DMSO, $400 \mathrm{MHz}$ ) $\delta 0.23(\mathrm{~s}$, $9 \mathrm{H}), 1.36(\mathrm{~s}, 9 \mathrm{H}), 6.01(\mathrm{q}, \mathrm{J}=7.2 \mathrm{~Hz}, 1 \mathrm{H}), 7.43(\mathrm{~d}, \mathrm{~J}=1.2 \mathrm{~Hz}$, $1 \mathrm{H}), 7.49(\mathrm{~d}, \mathrm{~J}=1.2 \mathrm{~Hz}, 1 \mathrm{H}), 7.84(\mathrm{~s}, 1 \mathrm{H})$; HRMS calcd for $\mathrm{C}_{18} \mathrm{H}_{23} \mathrm{~F}_{3} \mathrm{O}_{3} \mathrm{Si} \mathrm{m} / \mathrm{z}$ 373.14413, found 373.14440.

8-methoxy-2-(trifluoromethyl)-6-(trimethylsilyl)-2Hchromene-3-carboxylic acid (5m): Yield 93\%; white solid; mp 181-183 ${ }^{\circ} \mathrm{C}$; $1 \mathrm{H}$ NMR (d6-DMSO, $\left.400 \mathrm{MHz}\right) \delta 0.25$ (s, 9H), 3.84 (s, 3H), 5.93 (q, J=7.2Hz, 1H), 7.17 (s, 1H), 7.21 $(\mathrm{s}, 1 \mathrm{H}), 7.85(\mathrm{~s}, 1 \mathrm{H}), 13.24$ (brs, 1H) ; HRMS calcd for $\mathrm{C}_{15} \mathrm{H}_{17} \mathrm{~F}_{3} \mathrm{O}_{4} \mathrm{Si} \mathrm{m} / \mathrm{z} 347.09210$, found 347.09234.

8-cyclohexyl-2-(trifluoromethyl)-6-(trimethylsilyl)2H-chromene-3-carboxylic acid (5n): Yield 94\%; white solid; mp 203-205 ${ }^{\circ} \mathrm{C}$; $1 \mathrm{H}$ NMR (d6-DMSO, $\left.400 \mathrm{MHz}\right) \delta$ $0.23(\mathrm{~s}, 9 \mathrm{H}), 1.23-1.53(\mathrm{~m}, 5 \mathrm{H}), 1.71-1.81(\mathrm{~m}, 5 \mathrm{H}), 5.93-$ $5.94(\mathrm{~m}, 1 \mathrm{H}), 7.39(\mathrm{~s}, 1 \mathrm{H}), 7.44(\mathrm{~s}, 1 \mathrm{H}), 7.85(\mathrm{~s}, 1 \mathrm{H})$; HRMS calcd for $\mathrm{C}_{20} \mathrm{H}_{25} \mathrm{~F}_{3} \mathrm{O}_{3} \mathrm{Si} \mathrm{m} / \mathrm{z}$ 399.15978, found 399.15989.

8-phenyl-2-(trifluoromethyl)-6-(trimethylsilyl)-2Hchromene-3-carboxylic acid (5o): Yield 99\%; white solid; mp 194-194 ${ }^{\circ} \mathrm{C}$; $1 \mathrm{H}$ NMR (d6-DMSO, $\left.400 \mathrm{MHz}\right) \delta 0.27$ (s, 9H), 5.91 (q, J=7.2Hz, 1H), 7.34-7.39 (m, 1H), 7.43-7.49 (m, $5 \mathrm{H}), 7.66(\mathrm{~d}, \mathrm{~J}=0.8 \mathrm{~Hz}, 1 \mathrm{H}), 7.85(\mathrm{~s}, 1 \mathrm{H}), 7.94(\mathrm{~s}, 1 \mathrm{H})$; HRMS calcd for $\mathrm{C}_{20} \mathrm{H}_{19} \mathrm{~F}_{3} \mathrm{O}_{3} \mathrm{Si} \mathrm{m} / \mathrm{z}$ 393.11283, found 393.11322 .

8-(4-fluorophenyl)-2-(trifluoromethyl)-6-(trimethylsilyl)2H-chromene-3-carboxylic acid (5p): Yield 99\%; white solid; mp 227-229 ${ }^{\circ} \mathrm{C}$; $1 \mathrm{H}$ NMR (d6-DMSO, $\left.400 \mathrm{MHz}\right) \delta$ $0.26(\mathrm{~s}, 9 \mathrm{H}), 5.92(\mathrm{q}, \mathrm{J}=7.2 \mathrm{~Hz}, 1 \mathrm{H}), 7.26-7.30(\mathrm{~m}, 2 \mathrm{H}), 7.45$ $(\mathrm{d}, \mathrm{J}=1.6 \mathrm{~Hz}, 1 \mathrm{H}), 7.50-7.53(\mathrm{~m}, 2 \mathrm{H}), 7.65(\mathrm{~d}, \mathrm{~J}=1.6 \mathrm{~Hz}, 1 \mathrm{H})$, $7.93(\mathrm{~s}, 1 \mathrm{H})$; HRMS calcd for $\mathrm{C}_{20} \mathrm{H}_{18} \mathrm{~F}_{4} \mathrm{O}_{3} \mathrm{Si} \mathrm{m} / \mathrm{z} 411.10341$, found 411.10356 .

8-(p-tolyl)-2-(trifluoromethyl)-6-(trimethylsilyl)-2Hchromene-3-carboxylic acid (5q): Yield 97\%; white solid; mp 166-168 ${ }^{\circ} \mathrm{C}$; $1 \mathrm{H}$ NMR (d6-DMSO, $\left.400 \mathrm{MHz}\right) \delta 0.26(\mathrm{~s}$, 9H), 2.35 (s, 3H), 5.87 (q, J=7.2Hz, 1H), 7.24-7.26 (m, 2H), 7.36-7.38 (m, 3H), $7.53(\mathrm{~s}, 1 \mathrm{H}), 7.70(\mathrm{~s}, 1 \mathrm{H})$; HRMS calcd for $\mathrm{C}_{21} \mathrm{H}_{21} \mathrm{~F}_{3} \mathrm{O}_{3} \mathrm{Si} \mathrm{m} / \mathrm{z} 407.12848$, found 407.12885.

\section{CONFLICT OF INTERESTS}

The author(s) confirm that this article content has no conflicts of interest. 


\section{ACKNOWLEDGEMENT}

Y. Z. is grateful to the Guangzhou Government and Guangzhou Institute of Biomedicine and Health (GIBH), Chinese Academy of Sciences (CAS) for the joint sponsorship of this work under the Drug Discovery Pipeline (DDP) grant.

\section{REFERENCES}

[1] Yukiji, S.; Hiroshi, H. 1H-2-Benzopyran-1-one Derivatives, Microbial Products with Pharmacological Activity. Relationship between Structure and Activity in 6-[[ 1(S)-(3(S),4-Dihydro-8hydroxy-1-oxo-1H-2-benzopyran-3-yl)-3-methylbutyl]-amino]-4( S ),5 (S )-dihydroxy-6-oxo-3( $\mathrm{S}$ ) -ammoniohexanoate. J. Med. Chem., 1983, 26, 1370-1374.

[2] Hajela, K.; Kapil, R. S. Synthesis and post-coital contraceptive activity of a new series of substituted 2,3-diaryl-2 $\mathrm{H}$-1-benzopyrans. Eur. J. Med. Chem., 1997, 32, 135-142.

[3] Tang, L.; Yu, J.; Leng, Y.; Feng, Y.; Yang, Y.; Ji, R. Synthesis and insulin-sensitizing activity of a novel kind of benzopyran derivative. Bioorg. Med. Chem. Lett., 2003, 13, 3437-3440.

[4] Fre'de'rick, R.; Robert, S.; Charlier, C.; Wouters, J.; Masereel, B.; Pochet, L. Mechanism-based thrombin inhibitors: design, synthesis, and molecular docking of a new selective 2-oxo-2H-1-benzopyran derivative. J. Med. Chem., 2007, 50, 3645-3650.

[5] El-Shaaer, H. M.; Foltinova, P.; Lacova, M.; Chovancova, J.; Stankovicova, H. Synthesis, antimicrobial activity and bleaching effect of some reaction products of 4-oxo-4H-benzopyran-3carboxaldehydes with aminobenzothiazoles and hydrazides. Farmaco, 1998, 53, 224-232.

[6] Okazaki, H.; Kishi, T.;Beppu, T.; Arima, K. A new antibiotic baciphelacin. J. Antibiot., 1975, 28, 717-719.

[7] Wang, J.; Carter, L. J.; Kiefer, J. R.; Kurumbail, R. G.; Pawlitz, J.; Brown, L. D.; Hartmann, S.; Graneto, J. M. J.; Seibert, K.; Talley,
J. J. The novel benzopyran class of selective cyclooxygenase-2 inhibitors-part I: the first clinical candidate. Bioorg. Med. Chem. Lett., 2010, 20, 7155-7158.

[8] Wang, J. L.; Limburg, D.; Graneto, M. J.; Springer, J.; Hamper, J. R.B.; Liao, S.; Pawlitz, J. L.; Kurumbail, R. G.; Maziasz, T. Talley, J. J.; Kiefer , J. R.; Carter, J. The novel benzopyran class of selective cyclooxygenase-2 inhibitors. Part 2: The second clinical candidate having a shorter and favorable human half-life. Bioorg. Med. Chem. Lett., 2010, 20, 7159-7163.

[9] Wang, J. L.; Aston, K.; Limburg, D.; Ludwig, C.; Hallinan, A. E.; Koszyk, F.; Hamper, B.; Brown, D.; Graneto, M.; Talley, J.; Maziasz, T.; Masferrer, J.; Carter, J. The novel benzopyran class of selective cyclooxygenase-2 inhibitors. Part III: the three microdose candidates. Bioorg. Med. Chem. Lett., 2010, 20, 7164-7168.

[10] Colvin, E. W. Silicon Reagents in Organic Synthesis. Academic Press, London, 1988, p 39.

[11] Rich, J. D. A New Route to Arylsilanes. J. Am. Chem. Soc., 1989, 111, 5886-5893.

[12] Atwell, W.; Bokerman, G. N. Transition metal catalyzed silylations. US Patent 3,772,347, 1973; Chem. Abstr., 1973, 80, 3625.

[13] Matsumoto, H.; Nagashima, S.; Yoshihiro, K.; Nagai, Y. Siliconcarbon bond formation by the reaction of disilanes with halobenzenes in the presence of tetrakis(triphenylphosphine)palladium(0). J. Organomet. Chem., 1975, 85, C1-C3.

[14] Matsumoto, H.; Yoshihiro, K.; Nagashima, S.; Watanabe, H.; Nagai, Y. Conversion of disilanes to functional monosilanes : Preparation of some nitrophenyltrimethylsilanes by tetrakis(triphenylphosphine)palladium $(\mathrm{O})$ catalyzed reactions of hexamethyldisilane with nitrohalobenzenes. J. Organomet. Chem., 1977, 128, 409-413.

[15] Matsumoto, H.; Shono, K.; Nagai, Y. The reaction of hexamethyldisilane with dihalonitrobenzenes in the presence of tetrakis(triphenylphosphine)palladium(0). Synthesis of bis(trimethylsilyl) nitrobenzenes and (trimethylsilyl)chloronitrobenzenes. J. Organomet. Chem., 1981, 208, 145-152.

[16] L. J. Gooßen.; A. S. Ferwanah. A Mild and Efficient Protocol for the Catalytic Silylation of Aryl Bromides. Synlett, 2000, 2000 (12), 1801-1803.

Received: May 24, 2013

(C) Zhang et al.; Licensee Bentham Open.

This is an open access article licensed under the terms of the Creative Commons Attribution Non-Commercial License (http://creativecommons.org/licenses/ by-nc/3.0/) which permits unrestricted, non-commercial use, distribution and reproduction in any medium, provided the work is properly cited. 